\title{
Teleparallel dark energy model with a fermionic field via Noether symmetry
}

\author{
Yusuf Kucukakca ${ }^{\mathrm{a}}$ \\ Department of Physics, Faculty of Science, Akdeniz University, 07058 Antalya, Turkey
}

Received: 7 July 2014 / Accepted: 16 September 2014 / Published online: 30 September 2014

(c) The Author(s) 2014. This article is published with open access at Springerlink.com

\begin{abstract}
In the present work, we consider a model with a fermionic field that is non-minimally coupled to gravity in the framework of teleparallel gravity. In order to determine the forms of the coupling and potential function of fermionic field for the considered model, we use the Noether symmetry approach. By applying this approach, for the FriedmanRobertson-Walker metric, we obtain the respective potential and coupling functions as a linear and power-law form of the bilinear $\Psi$. Furthermore, we search for the exact cosmological solution of the model. It is shown that the fermionic field plays the role of dark energy.
\end{abstract}

\section{Introduction}

In modern cosmology, it is widely accepted that cosmic acceleration, called inflation, occurred in the very early universe prior to both radiation- and matter-dominated epochs. The idea of inflation was originally proposed in the early 1980 s by Guth [1] to solve several cosmological puzzles such as the flatness and horizon puzzles. After the radiation and matter-dominated epochs where the universe is in a decelerated expansion phase, as indicated by recent astrophysical observations of the type Ia supernovae [2-4] and cosmic microwave background radiation $[5,6]$, another cosmic acceleration occurred in the late-time universe. The source for this late-time acceleration was dubbed dark energy; its origin has not been identified yet although several candidates occur in the literature. The simplest candidate for the dark energy is the cosmological constant or the vacuum energy. Despite its agreement with the observational data, this model is facing the serious problems that the cosmological constant has (see for the review papers [7-10]).

In order to resolve the issue of the cosmic accelerated expansion of the universe, two approaches have been proposed. The first approach is to take a scalar or fermionic

\footnotetext{
a e-mail: ykucukakca@akdeniz.edu.tr
}

field as the matter content of the universe, constituting the right-hand side of the Einstein field equations. This approach includes a variety of scalar fields such as quintessence [11], phantom [12], quintom [13], tachyon [14], k-essence [15], or fermion fields [16-27]. The second approach is to modify the geometric part of the Einstein field equations. The $f(R)$ [29-32], Gauss-Bonnet [33], $f(R, T)$ [34], $f(T)$ gravities [35-39] are models belonging to the second approach.

The teleparallel theory of gravity, also a teleparallel equivalent of general relativity (GR), was propounded by Einstein with the aim of unifying gravity and electromagnetism $[40,41]$. Teleparallel theory is constructed by using the Weitzenbock connection, hence its Lagrangian density is described by a torsion scalar $T$ instead of the curvature scalar $R$ in GR, formulated with the Levi-Civita connection. In this theory, the dynamical variables represented by the four linearly independent vierbein (or tetrad) fields which play a similar role to the metric tensor in GR. The field equations of teleparallel gravity are obtained by taking the variation of the action with respect to the vierbein fields [42]. Recently, an interesting modified gravity by extending teleparallel theory, so-called $f(T)$ gravity, has been proposed to explain the current accelerating expansion of the universe without introducing the matter component [35-39]. In the recent literature, to check whether $f(T)$ gravity might be an alternative gravitational theory to the general relativity, its various properties have been investigated. We refer the reader to e.g. [43-63] for some relevant works. Another extension of teleparallel gravity can be made by introducing a scalar field which is non-minimally coupled to the torsion scalar. This can be regarded as a scalar-teleparallel theory of gravity, a modification of teleparallel gravity analogous to the scalartensor theory as a modification of the GR. That has recently been proposed as an alternative dark energy model [64-75]. The theory was called "teleparallel dark energy". It has been found that such a theory has a richer structure than the same one in the framework of general relativity. The richer struc- 
ture of a non-minimally coupled scalar field with the torsion scalar is due to exhibiting a quintessence-like or phantomlike behavior, or experiencing the phantom divide crossing in this theory. We note that in the minimal coupling case, the cosmological model with the quintessence scalar field in teleparallel gravity is identical to that in the GR.

On the other hand, some cosmological models were also investigated in the literature by considering fermionic fields (Dirac, or spinor field) as sources of the gravitational field in the framework of GR. In this sense, to describe both earlytime inflation and late-time acceleration of the universe, the models have been proposed by using the dynamics of fermion fields with suitable interaction potentials, where the fermion fields play the role of the inflaton or dark energy [16-27]. Note that in these works the fermionic field is a classical fermion field, presented in detail in [17]. Recently, we have also studied the fermionic fields as a source of inflation and dark energy in a $2+1$ dimensional gravity [28]. In addition, some cosmological solutions have been examined in the presence of a fermionic field in gravitational theories with a non-vanishing torsion [76] and $f(T)$ gravity [77].

In the present study, motivated by the teleparallel dark energy scenario and the role of the fermionic field in the cosmological context, we propose a fermionic teleparallel dark energy model in which the fermionic field with a potential non-minimally couples to the torsion scalar. Note that the model is completely equivalent to the standard GR when the fermion field is minimally coupled to the torsion scalar [21]. In such a model, we need to determine the forms of the coupling function $F(\Psi)$ and the potential $V(\Psi)$. The Noether symmetry approach introduced by de Rittis et al. and Cappoziello et al. allows one to determine the potential and the coupling function dynamically in scalar-tensor gravity theory [78-81]. Utilizing this approach, we find the potential and the coupling function in the teleparallel dark energy scenario with the fermionic field. We analytically solve the field equations of the theory evolving in a spatially flat Friedmann-Robertson-Walker spacetime. Our results show that the fermionic teleparallel dark energy equation of state parameter has both a quintessence and a phantom phase in this theory.

The structure of this paper is the following. In Sect. 2, the field equations are derived from a point-like Lagrangian in a Friedman-Robertson-Walker (FRW) spacetime, which is obtained from an action including the fermionic field nonminimally coupled to the torsion scalar in the framework of teleparallel gravity. In Sect. 3, we search for the Noether symmetry of the Lagrangian of the theory and in Sect. 4, we give the exact solutions of the field equations by using the coupling function and the potential obtaining the Noether symmetry approach. Finally, in Sect. 5, we conclude with a brief summary of the obtained results. It should be noted that we fully adopt the natural system of units by taking
$8 \pi G=c=\hbar=1$. Indices $i, j, l$ run from 1 to 4 throughout this paper.

\section{The action and the field equations}

The model considered in this work is described by the action for a fermion field that is non-minimally coupled with the torsion scalar,

$$
\begin{aligned}
\mathcal{A}= & \int \mathrm{d}^{4} x e\left\{F(\Psi) T+\frac{l}{2}\left[\bar{\psi} \Gamma^{\mu}\left(\overrightarrow{\partial_{\mu}}-\Omega_{\mu}\right) \psi\right.\right. \\
& \left.\left.-\bar{\psi}\left(\overleftarrow{\partial_{\mu}}+\Omega_{\mu}\right) \Gamma^{\mu} \psi\right]-V(\Psi)\right\},
\end{aligned}
$$

where $e=\operatorname{det}\left(e_{\mu}^{a}\right)=\sqrt{-g} ; e_{\mu}^{a}$ is a tetrad (vierbein) basis, $T$ is a torsion scalar, and $\psi$ and $\bar{\psi}=\psi^{\dagger} \gamma^{0}$ denote the spinor field and its adjoint, with the dagger representing complex conjugation. $F(\Psi)$ and $V(\Psi)$ are generic functions, representing the coupling with gravity and the self-interaction potential of the fermionic field, respectively. In this study, since we focus on the effect of the fermionic field in the context of teleparallel gravity, we can neglect the contribution of the ordinary matter. We note that the action in (1) with the torsion formulation of general relativity including the fermionic field is completely equivalent to the standard general relativity with the fermionic field minimally coupled to the Ricci scalar. In our study, for simplicity, we assume that $F$ and $V$ depend only on functions of the bilinear $\Psi=\bar{\psi} \psi$. In the above action, furthermore, $\Omega_{\mu}$ is the spin connection $\Omega_{\mu}=-\frac{1}{4} g_{\sigma v}\left[\Gamma_{\mu \lambda}^{v}-e_{b}^{v} \partial_{\mu} e_{\lambda}^{b}\right] \Gamma^{\sigma} \Gamma^{\lambda}$ with $\Gamma_{\mu \lambda}^{v}$ denoting the standard Levi-Civita connection and $\Gamma^{\mu}=e_{a}^{\mu} \gamma^{a}$. The $\gamma^{\mu}$ are the Dirac matrices.

We will consider here the simplest homogeneous and isotropic cosmological model, FRW, whose spatially flat metric is given by

$\mathrm{d} s^{2}=\mathrm{d} t^{2}-a^{2}(t)\left[\mathrm{d} x^{2}+\mathrm{d} y^{2}+\mathrm{d} z^{2}\right]$,

where $a(t)$ is the scale factor of the universe. In the teleparallel gravity, the torsion scalar corresponding to the FRW metric (2) takes the form of $T=-\frac{6 \dot{a}^{2}}{a^{2}}$, where the dot represents differentiation with respect to cosmic time $t$ (see [35]). Considering the background in (2), it is possible to obtain the point-like Lagrangian from the action (1)

$L=6 F a \dot{a}^{2}-\frac{l a^{3}}{2}\left(\bar{\psi} \gamma^{0} \dot{\psi}-\dot{\bar{\psi}} \gamma^{0} \psi\right)+a^{3} V$

here, because of the homogeneity and the isotropy of the metric it is assumed that the spinor field depends only on time, i.e. $\psi=\psi(t)$. The Dirac equations for the spinor field $\psi$ and its adjoint $\bar{\psi}$ are obtained from the point-like Lagrangian (3) 
such that the Euler-Lagrange equations for $\psi$ and $\bar{\psi}$ are

$\dot{\bar{\psi}}+\frac{3}{2} H \bar{\psi}-\imath\left(6 F^{\prime} H^{2}+V^{\prime}\right) \bar{\psi} \gamma^{0}=0$,

$\dot{\psi}+\frac{3}{2} H \psi+\imath\left(6 F^{\prime} H^{2}+V^{\prime}\right) \gamma^{0} \psi=0$,

where $H=\dot{a} / a$ denotes the Hubble parameter and the prime denotes a derivative with respect to the bilinear $\Psi$. On the other hand, from the point-like Lagrangian (3) and by considering the Dirac equations, we find the acceleration equation from the Euler-Lagrange equation for $a$,

$\frac{\ddot{a}}{a}=-\frac{\rho_{f}+3 p_{f}}{12 F}$.

Finally, we also have to consider the Hamiltonian constraint equation $\left(E_{L}=0\right)$ associated with the Lagrangian (3)

$E_{L}=\frac{\partial L}{\partial \dot{a}} \dot{a}+\frac{\partial L}{\partial \dot{\psi}} \dot{\psi}+\dot{\bar{\psi}} \frac{\partial L}{\partial \dot{\bar{\psi}}}-L$,

which yields the Friedmann equation as follows:

$H^{2}=\frac{\rho_{f}}{6 F}$.

In the acceleration and Friedmann equations, $\rho_{f}$ and $p_{f}$ are the effective energy density and pressure of the fermion field, respectively, so that they have the following forms:

$\rho_{f}=V$,

$p_{f}=4 F^{\prime} H \dot{\Psi}+\left(6 F^{\prime} H^{2}+V^{\prime}\right) \Psi-V$.

It is very hard to find a solution for (4)-(8) since these are highly non-linear systems. In order to solve the field equations we have to determine a form for the coupling function and the potential density of the theory. To do this, in the following section we will use the Noether symmetry approach.

\section{The Noether symmetry approach}

Symmetries play an important role in theoretical physics. Specially, symmetries of the Lagrangian, the so-called Noether symmetries, can be used to obtain the conserved quantities or constants of motion. The Noether symmetry approach tells us that the Lie derivative of the Lagrangian with respect to a given vector field $\mathbf{X}$ vanishes, i.e.

$£_{\mathbf{X}} L=0$.

If the condition (11) is satisfied, then $\mathbf{X}$ is said to be a symmetry for the dynamics derived from the Lagrangian $L$ and thus generates a conserved quantity. In fact, the idea of the application of the Noether symmetries as a cosmological tool is not new. It has been introduced by de Ritis et al. [78,79] and Capozziello et al. [80,81], in order to get solutions of the field equations in gravitational theories. We also note that such a technique helps us to find the coupling and potential function, restricting the arbitrariness in a suitable way in the non-minimal coupled scalar-tensor theories [82-88]. Some cosmological solutions have been presented both in the metric and Palatini $f(R)$ theory following the Noether symmetry approach [89-96]. The Noether symmetry approach is used to obtain exact forms of gravitational theories including $f(T)$ gravity in the literature [97-104]. On the other hand, some authors studied a cosmological model in the framework of GR where a spinor field is non-minimally coupled with the gravitational field via the Noether symmetry approach [21]. They determined the coupling and potential density of the spinor field and showed that the spinor field behaves as an inflaton describing an accelerated inflationary scenario. We will search for the Noether symmetries for our model. In terms of the components of the spinor field $\psi=\left(\psi_{1}, \psi_{2}, \psi_{3}, \psi_{4}\right)^{T}$ and its adjoint $\bar{\psi}=\left(\psi_{1}^{\dagger}, \psi_{2}^{\dagger},-\psi_{3}{ }^{\dagger},-\psi_{4}^{\dagger}\right)$, the Lagrangian (3) can be rewritten as

$L=6 F a \dot{a}^{2}-\frac{l a^{3}}{2} \sum_{i=1}^{4}\left(\psi_{i}^{\dagger} \dot{\psi}_{i}-\dot{\psi_{i}^{\dagger}} \psi_{i}\right)+a^{3} V$.

Now we seek the condition for the Lagrangian (12) to admit a Noether symmetry. The configuration space of this Lagrangian is $Q=\left(a, \psi_{j}, \psi_{j}^{\dagger}\right)$, whose tangent space is $T Q=\left(a, \psi_{j}, \psi_{j}^{\dagger}, \dot{a}, \dot{\psi}_{j}, \dot{\psi}_{j}^{\dagger}\right)$. The existence of the Noether symmetry given by (11), which implies the existence of a vector field $\mathbf{X}$ such that

$$
\begin{aligned}
\mathbf{X}= & \alpha \frac{\partial}{\partial a}+\dot{\alpha} \frac{\partial}{\partial \dot{a}} \\
& +\sum_{j=1}^{4}\left(\beta_{j} \frac{\partial}{\partial \psi_{j}}+\dot{\beta_{j}} \frac{\partial}{\partial \dot{\psi}_{j}}+\gamma_{j} \frac{\partial}{\partial \psi_{j}^{\dagger}}+\dot{\gamma_{j}} \frac{\partial}{\partial \dot{\psi_{j}^{\dagger}}}\right)
\end{aligned}
$$

where $\alpha, \beta_{j}$ and $\gamma_{j}$ are unknown functions of the variables $a, \psi_{j}$ and $\psi_{j}^{\dagger}$. Hence the Noether condition (11) leads to the following differential equations consisting of the coupled system of 19 equations:

$$
\begin{aligned}
& \alpha+2 a \frac{\partial \alpha}{\partial a}+\frac{F^{\prime}}{F} a \sum_{i=1}^{4}\left(\epsilon_{i} \beta_{i} \psi_{i}^{\dagger}+\epsilon_{i} \gamma_{i} \psi_{i}\right)=0, \\
& F \frac{\partial \alpha}{\partial \psi_{j}}=0, \quad F \frac{\partial \alpha}{\partial \psi_{j}^{\dagger}}=0, \\
& 3 \alpha \psi_{j}+a \beta_{j}-a \sum_{i=1}^{4}\left(\frac{\partial \beta_{i}}{\partial \psi_{j}^{\dagger}} \psi_{i}^{\dagger}-\frac{\partial \gamma_{i}}{\partial \psi_{j}^{\dagger}} \psi_{i}\right)=0,
\end{aligned}
$$


$3 \alpha \psi_{j}^{\dagger}+a \gamma_{j}+a \sum_{i=1}^{4}\left(\frac{\partial \beta_{i}}{\partial \psi_{j}} \psi_{i}^{\dagger}-\frac{\partial \gamma_{i}}{\partial \psi_{j}} \psi_{i}\right)=0$,

$\sum_{i=1}^{4}\left(\frac{\partial \beta_{i}}{\partial a} \psi_{i}^{\dagger}-\frac{\partial \gamma_{i}}{\partial a} \psi_{i}\right)=0$

$3 \alpha V+a V^{\prime} \sum_{i=1}^{4}\left(\epsilon_{i} \beta_{i} \psi_{i}^{\dagger}+\epsilon_{i} \gamma_{i} \psi_{i}\right)=0$,

where $\epsilon_{i}=\left\{\begin{array}{ll}1 & \text { for } i=1,2 \\ -1 & \text { for } i=3,4\end{array}\right.$. This system, given by (14)-(19), is obtained by imposing the fact that the coefficients of $\dot{a}^{2}, \dot{a}, \dot{\psi}_{j}, \dot{\psi}_{j}^{\dagger}, \dot{a} \dot{\psi}_{j}$ and $\dot{a} \dot{\psi}_{j}^{\dagger}$ vanish.

One can see from (15) that the coefficient $\alpha$ is only a function of $a$. From (19) one can rewrite it as follows:

$\frac{3 \alpha V}{a V^{\prime}}=-\sum_{i=1}^{4}\left(\epsilon_{i} \beta_{i} \psi_{i}^{\dagger}+\epsilon_{i} \gamma_{i} \psi_{i}\right)$.

We put (20) into (14) and, recalling that $F$ and $V$ are only functions of $\Psi$, the corresponding result is

$\frac{\alpha}{a} \frac{\partial \alpha}{\partial a}=\frac{3 F^{\prime} V}{2 F V^{\prime}}-\frac{1}{2}=n$,

where $n$ is a constant. Then we find $\alpha$ from (21)

$\alpha=\alpha_{0} a^{n}$,

where $\alpha_{0}$ is an integration constant. Now, from (17), (18), and (19), after some algebraic calculations, one can obtain the solutions for the other symmetry generators $\beta_{j}$ and $\gamma_{j}$ as follows:

$\beta_{j}=-\left(\frac{3}{2} \alpha_{0} a^{n-1}+\epsilon_{j} \beta_{0}\right) \psi_{j}$,

$\gamma_{j}=-\left(\frac{3}{2} \alpha_{0} a^{n-1}-\epsilon_{j} \beta_{0}\right) \psi_{j}^{\dagger}$,

where $\beta_{0}$ is a constant of integration. Using the above solution in (20) and (21), the potential $U(\Psi)$ and the coupling function $F(\Psi)$ are obtained,

$V(\Psi)=\lambda \Psi$,

$F(\Psi)=f_{0} \Psi^{\frac{2 n+1}{3}}$

where $\lambda$ and $f_{0}$ are constants.

For $n=-1 / 2$, the coupling function given by (25) becomes constant, so that our model is reduced to an action which contains a fermion field that is minimally coupled with the torsion scalar. Such a selection of the Noether symmetry condition for the potential function given by (24) yields a free Dirac spinor field with a mass term. Therefore, one can consider the mass term $m$ instead of $\lambda$. In the next section we shall search cosmological solutions of the field equations using the obtained coupling functions $F(\Psi)$ and the potential $V(\Psi)$.

\section{Exact cosmological solutions}

In this section, we attempt to integrate the dynamical system given by (4)-(8) analytically. Since the coupling and potential functions depend on the bilinear function $\Psi$, using the Dirac equations (4) and (5) one gets

$\dot{\Psi}+3 \frac{\dot{a}}{a} \Psi=0$,

and integration gives

$\Psi=\frac{\Psi_{0}}{a^{3}}$,

where $\Psi_{0}$ is a constant of integration. We note that, since the field equations can be directly integrable, it is not necessary to calculate the constants of motion associated with the Noether symmetry. Also the constants of motion give no new constraint on the field equations. From the above solution, the acceleration and Friedmann equations become only a function of the cosmic scale factor, and they can be directly integrated as indicated in the following cases.

\subsection{Case A}

Firstly, we consider the fermion field to be minimally coupled to the torsion scalar, i.e. $n=-1 / 2$. This case has been studied in [21]. Using the potential (24) in the Friedmann equation together with (9), the time evolution of the scale factor can easily be calculated and has the form

$a(t)=\left(\frac{3 \lambda \Psi_{0}}{4}\right)^{1 / 3}\left(t-c_{1}\right)^{2 / 3}$,

here $c_{1}$ is an integration constant and we take $f_{0}=\frac{1}{2}$. The energy density and pressure of the fermionic field follow from (9) and (10), yielding

$\rho_{f}=\frac{4}{3\left(t-c_{1}\right)^{2}}, \quad p_{f}=0$.

Therefore, from these solutions we conclude that the fermionic field behaves as a standard pressureless matter field.

\subsection{Case B}

Now, we consider the general case where the coupling function is $F(\Psi)=f_{0} \Psi^{\frac{2 n+1}{3}}$. The Friedmann equation for this case can be rewritten as

$\dot{a}^{2}=a_{0} a^{2 n}, \quad a 0=\frac{\lambda \Psi_{0}^{\frac{2(1-n)}{3}}}{6 f_{0}}$.

The general solution of the equation is

$a(t)=\left[a_{0}(n-1)\left(t-c_{2}\right)\right]^{-\frac{1}{n-1}}$, 
where $c_{2}$ is another integration constant and $n \neq 1$. Inserting the solution (31) into the acceleration equation (6) together with (9) and (10), we get $\lambda=6 f_{0}$. For $n=1$, the coupling function is reduced to the form $F(\Psi)=f_{0} \Psi$, so that the solution of (31) for the cosmic scale factor can be obtained by $a(t)=c_{3} \exp \left(\sqrt{a_{0}} t\right)$, which stands for a de Sitter solution. Thus, this solution shows that the fermionic field can behave as an inflaton.

The deceleration parameter, which is an important quantity in cosmology, is defined by $q=-a \ddot{a} / \dot{a}^{2}$, where the positive sign of $q$ indicates the standard decelerating models and the negative sign corresponds to accelerating models. The case $q=0$ corresponds to expansion with a constant velocity. It takes the following form in this model:

$q=-n$

From (32) we see that the universe is accelerating for $n>0$ and decelerating for $n<0$. We can also define the equation of state parameter for the fermionic field by using (6)-(10): $w_{f} \equiv \frac{P_{f}}{\rho_{f}}=\frac{2 q-1}{3}$. Then it can be obtained by

$w_{f}=-\frac{2 n+1}{3}$,

where the time evolution of the energy density and pressure of the fermion field read

$\rho_{f}=\lambda \Psi_{0}\left[a_{0}(n-1)\left(t-c_{2}\right)\right]^{\frac{3}{n-1}}=-\frac{2 n+1}{3} p_{f}$.

Cosmological observations show that $w$ lies in a very narrow strip close to $w=-1$. The case $w=-1$ corresponds to the cosmological constant. For $w<-1$, the phantom phase is observed, and for $-1<w<-1 / 3$ the phase is described by quintessence. Thus, in the interval $0<n<1$, we have the quintessence phase. If $n>1$, then the phantom phase occurs, where the universe is both expanding and accelerating. Therefore, we conclude that the fermionic field behaves as both the quintessence and the phantom dark energy.

\section{Conclusions}

Teleparallel gravity is an equivalent formulation of GR in which, instead of the curvature scalar $R$, one utilizes the torsion scalar $T$ for the action. By extending teleparallel gravity, some authors have recently suggested teleparallel dark energy models to explain the cosmic acceleration of the universe [64-75]. That was also our motivation in the present study where we proposed a new teleparallel dark energy model in which a fermionic field has a potential and it also is non-minimally coupled to gravity in the framework of teleparallel gravity. The Noether symmetry approach is useful in obtaining physically viable choices of the coupling and potential function of the fermionic field. By applying this approach to the Lagrangian given by (12), we have obtained the explicit forms of the corresponding coupling and potential function as $V(\Psi)=\lambda \Psi$ and $F(\Psi)=f_{0} \Psi^{\frac{2 n+1}{3}}$, respectively. For the minimally coupled fermion field case, which is equivalent to GR, i.e. for $n=-1 / 2$, the cosmological solution shows that the fermionic field behaves like a standard pressureless matter field. On the other hand, in the nonminimally coupled fermion field case, for $n=1$ we found the de Sitter solution, whereas for general $n$ we found the power law expansion for the cosmological scale factor [see Eq. (31)]. We have also presented the equation of state parameter of the fermionic field for our model. It turned out that a phantom-like dark energy for the intervals $0<n<1$ and a quintessence-like dark energy for the interval $n>1$ occur. Thus an important consequence of this work is that the fermionic field may be interpreted as a source of dark energy.

Finally, in the framework of GR, it is important to emphasize that when a fermionic field is non-minimally coupled to gravity, the existence of Noether symmetry yields only a cosmologically solution that describes the early-time accelerated expansion (see [21]). But in the framework of teleparallel gravity, this symmetry yields cosmological solutions that describe not only the early-time but also late-time accelerated expansion.

Acknowledgments We are grateful to Dr. Yusuf Sucu and Dr. Timur Sahin for fruitful discussions. This work was supported by Akdeniz University, Scientific Research Projects Unit.

Open Access This article is distributed under the terms of the Creative Commons Attribution License which permits any use, distribution, and reproduction in any medium, provided the original author(s) and the source are credited.

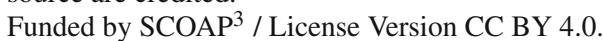

\section{References}

1. A.H. Guth, Phys. Rev. D 23, 347 (1981)

2. A.G. Riess et al., Astrophys. J. 116, 1009-1038 (1998)

3. S. Perlmutter et al., Astrophys. J. 517, 565-586 (1999)

4. D.N. Spergel et al., Astrophys. J. Suppl. 148, 213 (2003)

5. C.B. Netterfield et al., Astrophys. J. 571, 664 (2002)

6. C.N. Bennett et al., Astrophys. J. Suppl. 148, 1 (2003)

7. V. Sahni, A.A. Starobinsky, Int. J. Mod. Phys. D 9, 373 (2000)

8. S.M. Carroll, Living Rev. Relativ. 4, 1 (2001)

9. P.J.E. Peebles, B. Ratra, Rev. Mod. Phys. 75, 559 (2003)

10. E.J. Copeland, M. Sami, S. Tsujikawa, Int. J. Mod. Phys. D. 15, 1753-1935 (2006)

11. B. Ratra, P.J.E. Peebles, Phys. Rev. D 37, 3406 (1988)

12. R.R. Caldwell, Phys. Lett. B 545, 23 (2002)

13. M.Z. Li, B. Feng, X.M. Zhang, J. Cosmol. Astropart. Phys. 512, $002(2005)$

14. A. Sen, J. High Energy Phys. 04, 048 (2002)

15. C. Armendáriz-Picón, V. Mukhanov, Phys. Rev. D 63, 103510 (2001) 
16. B. Saha, Phys. Rev. D 64, 123501 (2001)

17. C. Armendáriz-Picón, P.B. Greene, Gen. Relativ. Gravit. 35, 1637 (2003)

18. B. Saha, T. Boyadjiev, Phys. Rev. D 69, 124010 (2004)

19. M.O. Ribas, F.P. Devecchi, G.M. Kremer, Phys. Rev. D 72, 123502 (2005)

20. M.O. Ribas, F.P. Devecchi, G.M. Kremer, Europhys. Lett. 81, 19001 (2008)

21. R.C. de Souza, G.M. Kremer, Class. Quantum Gravity 25, 225006 (2008)

22. B. Vakili, S. Jalalzadeh, H.R. Sepangi, J. Cosmol. Astropart. Phys. 05, 006 (2005)

23. L.L. Samojeden, F.P. Devecchi, G.M. Kremer, Phys. Rev. D 81, 027301 (2010)

24. R. Rakhi, G.V. Vijayagovindan, K. Indulekha, N.P. Abraham, Mod. Phys. A 25, 1267-1278 (2010)

25. D.-J. Liu, Phys. Rev. D 82, 063523 (2010)

26. R.C. de Souza, G.M. Kremer, Class. Quantum Gravity 28, 125006 (2011)

27. Y.A. Rybalov, A.N. Makarenko, K.N. Osetrin, Astrophys. Space Sci. 349, 561-566 (2014)

28. G. Gecim, Y. Kucukakca, Y. Sucu, Noether symmetries of Dirac field in $2+1$ dimensional gravity (2014). (Submitted for publication)

29. T.P. Sotiriou, V. Faraoni, Rev. Mod. Phys. 82, 451 (2010)

30. A. De Felice, S. Tsujikawa, Living Rev. Relativ. 13, 3 (2010)

31. S. Nojiri, S.D. Odintsov, Phys. Rep. 505, 59 (2011)

32. S. Capozziello, M. De Laurentis, Phys. Rep. 509, 167 (2011)

33. S. Nojiri, S.D. Odintsov, M. Sasaki, Phys. Rev. D 71, 123509 (2005)

34. T. Harko, F.S.N. Lobo, S. Nojiri, S.D. Odintsov, Phys. Rev. D 84, 024020 (2011)

35. G.R. Bengochea, R. Ferraro, Phys. Rev. D 79, 124019 (2009)

36. E. Linder, Phys. Rev. D 81, 127301 (2010)

37. K. Bamba, C.-Q. Geng, C.-C. Lee (2010). arXiv:1008.4036 [astroph.CO]

38. P. Wu, H.W. Yu, Phys. Lett. B 693, 415-420 (2010)

39. R. Myrzakulov, Eur. Phys. J. C 71, 1752 (2011)

40. A. Einstein, Sitzber. Preuss. Akad. Wiss. Phys. Math. K1. 217 (1928)

41. A. Einstein, Sitzber. Preuss. Akad. Wiss. Phys. Math. Kl. 224 (1928)

42. K. Hayashi, T. Shirafuji, Phys. Rev. D 19, 3524 (1979)

43. R. Zheng, Q.-G. Huang, J. Cosmol. Astropart. Phys. 03, 002 (2011)

44. K. Bamba, C.-Q. Geng, C.-C. Lee, L.-W. Luo, J. Cosmol. Astropart. Phys. 01, 021 (2011)

45. T. Wang, Phys. Rev. D 84, 024042 (2011)

46. R.-J. Yang, Europhys. Lett. 93, 60001 (2011)

47. G.R. Bengochea, Phys. Lett. B 695, 405 (2011)

48. P. Wu, H.W. Yu, Phys. Lett. B 692, 176 (2010)

49. B. Li, T.P. Sotiriou, J.D. Barrow, Phys. Rev. D 83, 064035 (2011)

50. Y. Zhang, H. Li, Y. Gong, Z.-H. Zhu, J. Cosmol. Astropart. Phys. 07, 015 (2011)

51. C. Deliduman, B. Yapiskan (2010). arXiv:1103.2225 [gr-qc]

52. S. Chattopadhyay, U. Debnath, Int. J. Mod. Phys. D 20, 1135 (2011)

53. M. Sharif, S. Rani, Mod. Phys. Lett. A 26, 1657 (2011)

54. S. Capozziello, V. Cardone, H. Farajollahi, A. Ravanpak, Phys. Rev. D 84, 043527 (2011)

55. R. Ferraro, F. Fiorini, Phys. Rev. D 84, 083518 (2011)

56. M.H. Daouda, M.E. Rodrigues, M. Houndjo, Eur. Phys. J. C 72, $1890(2012)$

57. H. Farajollahi, A. Ravanpak, P. Wu, Astrophys. Space Sci. 338 , $23(2012)$
58. M. Jamil, D. Momeni, N. Serikbayev, R. Myrzakulov, Astrophys. Space Sci. 339, 37 (2012)

59. K. Karami, A. Abdolmaleki, J. Cosmol. Astropart. Phys. 04, 007 (2012)

60. M. Jamil, D. Momeni, R. Myrzakulov, Eur. Phys. J. C 72, 2122 (2012)

61. S. Capozziello, P.A. Gonzalez, E.N. Saridakis, Y. Vasquez, J. High Energy Phys. 2013, 039 (2013)

62. K. Karami, A. Abdolmaleki, S. Asadzadeh, Z. Safari, Eur. Phys. J. C 73, 2565 (2013)

63. M. Sharif, S. Rani, Astrophys. Space Sci. 346, 573-582 (2013)

64. C.-Q. Geng, C.-C. Lee, E.N. Saridakis, Y.-P. Wu, Phys. Lett. B 704, $384(2011)$

65. C.-Q. Geng, C.-C. Lee, E.N. Saridakis, J. Cosmol. Astropart. Phys. 01, 002 (2012)

66. H. Wei, Phys. Lett. B 712, 430 (2012)

67. C. Xu, E.N. Saridakis, G. Leond, J. Cosmol. Astropart. Phys. 07, 005 (2012)

68. A. Banijamali, B. Fazlpour, Astrophys. Space Sci. 342, 229-235 (2012)

69. J.A. Gu, C.-C. Lee, C.-Q. Geng, Phys. Lett. B 718, 722 (2013)

70. Y. Kucukakca, Eur. Phys. J. C 73, 2327 (2013)

71. H.M. Sadjadi, Phys. Rev. D 87, 064028 (2013)

72. G. Otalora, J. Cosmol. Astropart. Phys. 07, 044 (2013)

73. C.-Q. Geng, J.A. Gu, C.-C. Lee, Phys. Rev. D 88, 024030 (2013)

74. G. Otalora, Phys. Rev. D 88, 063505 (2013)

75. J.T. Li, Y.-P. Wu, C.-Q. Geng, Phys. Rev. D 89, 044040 (2014)

76. S. Carloni, S. Vignolo, L. Fabbri, Class. Quantum Gravity 30, 205010 (2013)

77. R. Myrzakulov, D. Saez-Gomez, P. Tsyba (2013). arXiv:1311.5261 [gr-qc]

78. R. de Ritis, G. Marmo, G. Platania, C. Rubano, P. Scudellaro, C Stornaiolo, Phys. Rev. D 42, 1091 (1990)

79. M. Demianski, R. de Ritis, C. Rubano, P. Scudellaro, Phys. Rev. D 46, 1391 (1992)

80. S. Capozziello, R. de Ritis, Phys. Lett. A 177, 1 (1993)

81. S. Capozziello, Lambiase. Gen. Relativ. Gravit. 32, 673 (2000)

82. A.K. Sanyal, Phys. Lett. B 524, 177 (2002)

83. U. Camci, Y. Kucukakca, Phys. Rev. D 76, 084023 (2007)

84. S. Basilakos, M. Tsamparlis, A. Paliathanasis, Phys. Rev. D 83, $103512(2011)$

85. Y. Kucukakca, U. Camci, I. Semiz, Gen. Relativ. Gravit. 44, 1893 (2012)

86. M. Sharif, S. Waheed, J. Cosmol. Astropart. Phys. 02, 043 (2013)

87. R.C. de Souza, R. André, G.M. Kremer, Phys. Rev. D 87, 083510 (2013)

88. A. Paliathanasis, M. Tsamparlis, S. Basilakos, S. Capozziello (2014). arXiv:1403.0332 [astro-ph.CO]

89. S. Capozziello, A. De Felice, J. Cosmol. Astropart. Phys. 08, 016 (2008)

90. B. Vakili, Phys. Lett. B 16, 664 (2008)

91. M. Roshan, F. Shojai, Phys. Lett. B 668, 238 (2008)

92. A. Paliathanasis, M. Tsamparlis, S. Basilakos, Phys. Rev. D 84, 123514 (2011)

93. I. Hussain, M. Jamil, F.M. Mahomed, Astrophys. Space Sci. 337, 373 (2012)

94. Y. Kucukakca, U. Camci, Astrophys. Space Sci. 338, 211 (2012)

95. M.F. Shamir, A. Jhangeer, A.A. Bhatti, Chin. Phys. Lett. 29, $080402(2012)$

96. F. Darabi, K. Atazadeh, A. Rezaei-Aghdam, Eur. Phys. J. C 73, 2657 (2013)

97. H. Wei, X.J. Guo, L.F. Wang, Phys. Lett. B 707, 298 (2012)

98. K. Atazadeh, F. Darabi, Eur. Phys. J. C 72, 2016 (2012)

99. M. Jamil, D. Momeni, R. Myrzakulov, Eur. Phys. J. C 72, 2137 (2012)

100. H.M. Sadjadi, Phys. Lett. B 718, 270 (2012) 
101. H. Dong, J. Wang, X. Meng, Eur. Phys. J. C 73, 2543 (2013)

102. A. Aslam, M. Jamil, R. Myrzakulov, Phys. Scr. 88, 025003 (2013)

103. S. Basilakos, S. Capozziello, M. De Laurentis, A. Paliathanasis, M. Tsamparlis, Phys. Rev. D 88, 103526 (2013)
104. A. Paliathanasis, S. Basilakos, E.N. Saridakis, S. Capozziello, K. Atazadeh, F. Darabi, M. Tsamparlis, Phys. Rev. D 89, 104042 (2014) 